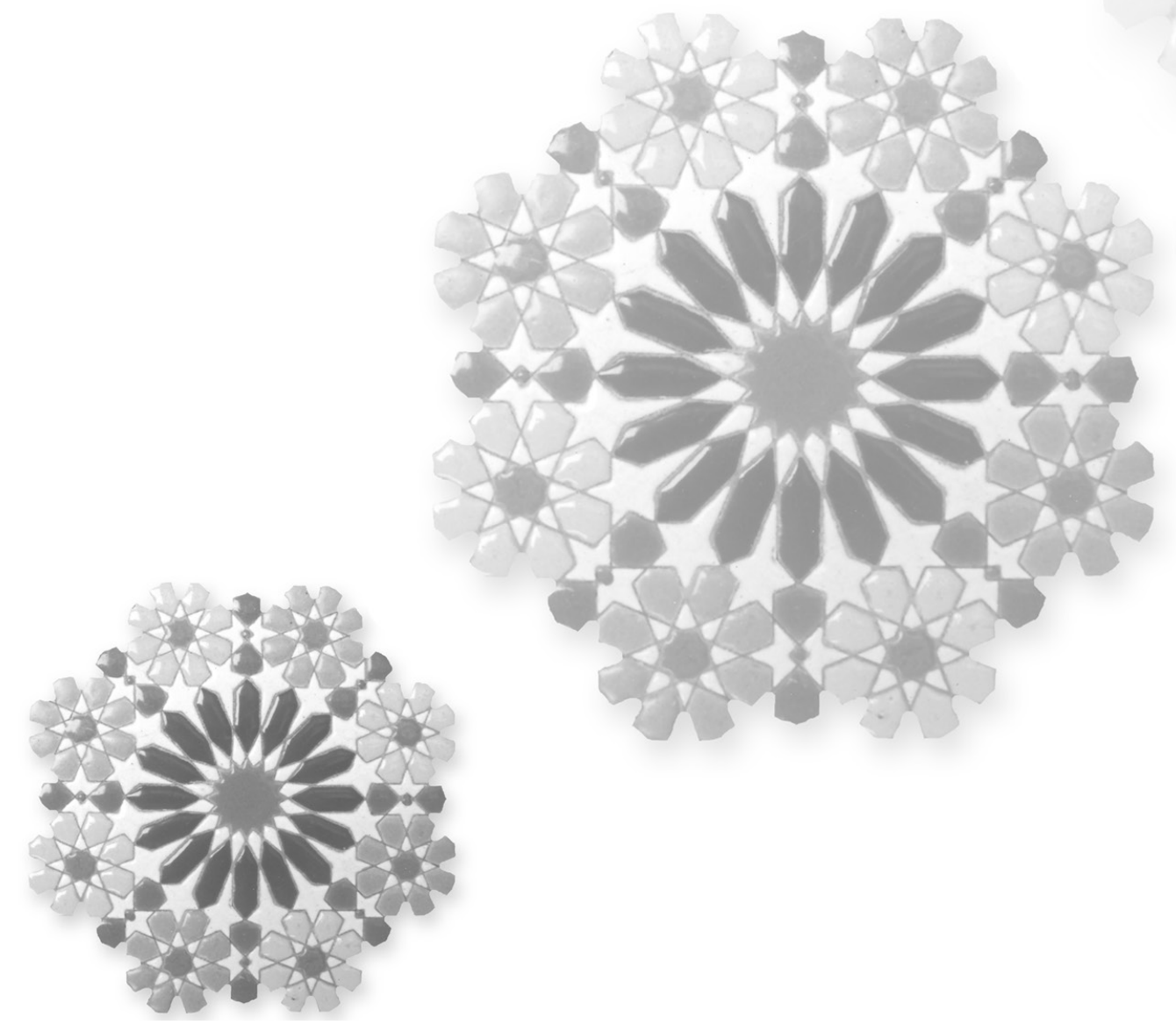




\section{Construcción de la} infancia y de un saber médico especializado: los comienzos de la pediatría en Buenos Aires, 1890-1920

\section{The construction of childhood and its specialized medical care: the beginnings of pediatrics in Buenos Aires, 1890-1920}

María Adelaida Colangelo

Investigadora, Centro de Estudios en Nutrición y Desarrollo Infantil/ Comisión de Investigaciones Científicas de la Provincia de Buenos Aires. La Plata - Buenos Aires - Argentina

adecolangelo@yahoo.com.ar

Recebido em 21 fev. 2017.

Aprovado em 10 jan. 2018.
COLANGELO, María Adelaida.

Construcción de la infancia y de un saber médico especializado: los comienzos de la pediatría en Buenos Aires, 1890-1920. História, Ciências, Saúde - Manguinhos, Rio de Janeiro, v.25, n.4, out.-dez. 2018, p.1219-1237.

\section{Resumen}

Se analiza la constitución de la pediatría como un saber médico especializado en Buenos Aires, entre 1890 y 1920 . Se busca mostrar que este proceso resulta de un doble movimiento: la delimitación y caracterización del niño como un particular objeto de conocimiento e intervención y la institucionalización de ciertos médicos como un grupo profesional que procura legitimar su especificidad tanto hacia el interior de la medicina como en el campo social más amplio. Luego de contextualizar socio históricamente las preocupaciones del saber médico acerca de la infancia, se profundiza en el modo en que ésta es construida, así como en el movimiento asociativo y académico que permite a los pediatras constituirse como un grupo de especialistas portadores de un saber experto.

Palabras clave: pediatría; especialización profesional; infancia; Buenos Aires.

\section{Abstract}

It analyzes the set-up of pediatrics as a specialized medical field in Buenos Aires, between 1890-1920. The article seeks to demonstrate this process as a result of two movements: the delimitation and characterization of children as a particular object of knowledge and intervention, and the institutionalization of some physicians as a professional group that sought to legitimate their specificity within the medicine field and beyond. After contextualizing socially and historically the concerns of medical knowledge on childhood, it explores with detail how the field was constructed, and the associative and academic movement that allowed pediatricians to constitute themselves as a group of specialists that bear a specific expertise.

Keywords: pediatrics; professional specialization; childhood; Buenos Aires. 


\begin{abstract}
Como em todos os outros níveis de organização da modernidade, também a biomedicina vai aos poucos - e desde muito cedo tendo que lidar com a reinstauração da Diferença. Seus saberes auxiliares ou particulares, a anatomia comparada, a biologia, a antropologia física, a psiquiatria, vão fornecendo os argumentos necessários à transposição para o mundo da 'corporalidade' de demarcações morais justificatórias de novas hierarquizações dos seres humanos (Duarte, 2001, p.8).
\end{abstract}

Como parte de un intenso proceso de medicalización de la vida en general y de la niñez en particular, en la Argentina de fines del siglo XIX, al igual que en otros países latinoamericanos, comienzan a configurarse nuevas especialidades médicas que delimitan nuevos objetos de estudio e intervención (Armus, 2005).

Con base en las etapas de la vida, pero también en la "raza", el "sexo" o en características socioculturales, la definición médica de la infancia puede ser considerada un ejemplo, entre otros, de la demarcación y clasificación de grupos sociales que las disciplinas científicas modernas establecen en su abordaje del fenómeno humano, tal como se señala en el epígrafe seleccionado. En el caso particular de la niñez, este proceso ha dado lugar a la configuración e institucionalización de la pediatría como especialidad o "rama" de la medicina. Ello implica reconocer no solo que la infancia, como categoría social específica, es resultado de procesos históricos relativamente recientes, ligados al advenimiento de la modernidad occidental (Ariès, 1981; Perrot, 1989) sino también que los saberes científicos - entre ellos la medicina - han desempeñado un papel clave en esas transformaciones.

A lo largo de este artículo se propone analizar algunos aspectos de la constitución de un saber médico especializado en la infancia en la Argentina de fines del siglo XIX y comienzos del XX, haciendo énfasis en la relación mutuamente constitutiva con su objeto de conocimiento. Partiendo de estudios que han explicado la profesionalización de la medicina en Argentina (González Leandri, 1996, 2006, 2012; Belmartino, 2005), se pone el foco en el proceso mediante el cual algunos médicos construyen una especialidad dentro de las ciencias médicas a partir de la especificidad atribuida a la niñez y a través de desarrollos académicos y asociativos que se dan de conjunto con el lugar de expertos que logran ocupar en el Estado. Este proceso de especialización profesional debe comprenderse en el marco de cambios sociales más amplios en la relación entre adultos y niños (Armstrong, 1986); cambios que implican el reconocimiento de la infancia como una categoría social específica y su construcción como un problema social en el marco de la preocupación por la población del Estado argentino recientemente organizado.

Esta indagación se realizó mediante el análisis discursivo de fuentes escritas, consistentes en textos escritos y/o debatidos por médicos que desempeñaron su profesión en Argentina entre 1890 y 1920, clasificadas en obras eruditas y de divulgación de acuerdo a la finalidad y los destinatarios para los que fueron producidas.

Se trabajó especialmente con las obras eruditas, destinadas al medio académico y profesional: artículos publicados en revistas científicas, libros, folletos científicos e informes de directores de establecimientos sanitarios. En este primer grupo de obras, el interés se centró en aquellos textos escritos por los médicos para presentar posiciones y discutir con 
sus pares $\mathrm{u}$ otros profesionales acerca de la especificidad de su abordaje de la infancia. Complementariamente se analizaron obras escritas para un público lego con la finalidad de divulgar los principios científicos de la crianza infantil: manuales, cartillas y revistas.

En el abordaje del tema confluyen los aportes de trabajos que analizan la construcción y el lugar social de la infancia en Argentina entre fines del siglo XIX y comienzos del XX, con las contribuciones de estudios centrados en la profesionalización de la medicina y su vinculación con las políticas públicas del Estado recientemente organizado. Entre los primeros, pueden mencionarse los análisis realizados por Ríos y Talak (1999), Cowen (2000), Zapiola (2009), así como los reunidos en las compilaciones de Lionetti y Míguez (2010) y Cosse et al. (2011). Estos trabajos permiten visualizar el lugar central que la infancia ocupa en la definición de problemas sociales en la época analizada, así como también la multiplicidad de actores e instituciones que participan en su categorización, clasificación y gobierno.

La profesionalización de la medicina en Argentina ha sido estudiada por González Leandri (1996, 2000, 2006, 2012; Belmartino, 2005; Álvarez, Carbonetti, 2008) y, en relación con la medicalización de la enfermedad y las políticas públicas ligadas al higienismo, por Armus (2005, 2010), Armus y Belmartino (2001) y Lobato (1996), entre otros. De una u otra manera estas investigaciones dan cuenta de la imposibilidad de trazar fronteras nítidas entre esferas profesionales, estatales, académicas y políticas, a la hora de pensar el quehacer y el lugar de los médicos en Buenos Aires en el periodo analizado (Soprano, 2014).

En la intersección del interés por el saber médico y por las políticas públicas de protección materno-infantil construidas entre fines del siglo XIX y las primeras décadas del XX pueden mencionarse trabajos como los de Biernat y Ramacciotti (2008, 2013), Billorou (2008) y otros como los de Nari (2004) y Di Liscia (2005) que hacen especial énfasis en el género, mostrando la relación entre el discurso médico higienista y el reforzamiento del papel materno atribuido a la mujer. No obstante, a excepción de los de Rustoyburu (2011, 2015) y Briolotti (2016), no son muchos los estudios con foco en la concepción de infancia y de sujeto infantil que contribuyen a construir los saberes médicos, ni que analizan las características de la conformación de la pediatría como saber especializado. Al respeto, tres trabajos sobre la constitución y características de la pediatría como especialidad médica en Brasil (Rivorêdo, 1998; Castellanos, 2003; Pereira, 2006) proporcionan valiosos elementos para pensar procesos semejantes en Argentina.

El periodo histórico delimitado para el análisis corresponde al momento en que comienza a ser construido, desde el Estado, un conjunto de discursos y prácticas destinadas a la infancia y a la maternidad, vinculados a las propuestas de la higiene y en relación con un problema social definido en términos de alta mortalidad infantil. Dicho periodo, no obstante, no puede ser considerado homogéneo, existiendo diferencias, que se visualizan en la primera posguerra, entre la organización incipiente de saberes e instituciones de sus comienzos, la conformación de la institucionalización estatal y una progresiva profesionalización de los recursos humanos de atención de la salud infantil (Nari, 2004; Di Liscia, 2005).

El trabajo comienza situando la profesionalización del saber médico en el marco de la definición de problemas sociales vinculados a la conformación de la población del Estado recientemente organizado; problemas en los que la infancia ocupa un lugar central en 
tanto "capital humano de la nación" afectado por altas tasas de mortalidad. Se abordan luego los procesos de constitución de la pediatría, enfocando primero el modo en que el niño es construido como un particular objeto de estudio e intervención, cuya atribuida especificidad permite a ciertos médicos apelar a la necesidad de un abordaje especializado. A partir de ello, se reconstruye el movimiento asociativo, académico y científico a través del cual quienes comienzan a autodenominarse como pediatras delimitan y buscan legitimar su especialidad en el marco de la medicina y del campo social más amplio. Por último, se aborda brevemente el modo en que la producción e institucionalización de ese saber especializado se vincula con los procesos estatales de construcción de políticas públicas. Por razones de espacio y por ser los aspectos menos explorados en otras investigaciones, gran parte del análisis se centra en los procesos de construcción epistemológica e institucional del nuevo colectivo profesional, a partir de los cuales éste busca ocupar un lugar de experto en los procesos de intervención estatal.

\section{Saber médico, infancia y cuestión social}

En Argentina, la constitución de la medicina como un campo científico profesional puede ser situada a partir de 1870. En términos de la delimitación, entendiendo este proceso de profesionalización por parte de los médicos diplomados como un espacio para la práctica legítima y monopólica del "arte de curar", González Leandri (1996, 2000) considera que los médicos en el Buenos Aires de la segunda mitad del siglo XIX ya conforman una profesión culta o erudita, una elite pequeña aunque influyente que depende fundamentalmente de una legitimación estatal. Tal como sucede en Europa y en el resto de Latinoamérica, se tornan hombres de peso en la vida cultural y política. Son considerados como expertos no solo en el campo médico, sino también en el social más general, apelando a la autoridad que les proporciona el desarrollo de la higiene para diseñar políticas públicas en el Estado recientemente organizado (Belmartino, 2005). De allí que González Leandri (2012), retomando las ideas de Plotkin y Zimmermann (2012), se refiera a un movimiento de doble construcción de la medicina como profesión y como un "saber del Estado".

Sustentando ese proceso, la población se constituye en objeto de análisis y de gestión estatal a través de políticas migratorias, sanitarias y educativas, en las que el cuidado de la infancia ocupa un lugar central. En efecto, los niños ya no solo son considerados valiosos para sus familias, sino en términos de "capital humano de la nación", un capital a cuidar, desarrollar y administrar.

Las asociaciones que se establecen entre riqueza, población y salud (González Leandri, 2000) enmarcan así el proyecto higienista que a partir del último tercio del siglo XIX se constituye como un amplio programa orientado a lograr la "salud biológica y social", centrado en un comienzo en el abordaje de las epidemias y los aspectos de la vida urbana que facilitan los contagios y, ya entrado el siglo XX, en el tratamiento de los problemas derivados de la llamada "cuestión social". Entendida como la preocupación acerca de la capacidad de mantener la cohesión social ante las consecuencias indeseadas de la modernización capitalista y la inmigración masiva, la "cuestión social" se expresa en la construcción social de un conjunto de problemas asociados a la pobreza urbana (hacinamiento, delito, 
insalubridad) y remite a procesos que atraviesan las grandes ciudades de la Argentina de la época, especialmente Buenos Aires y Rosario. ${ }^{1}$ Cabe señalar que en el período histórico considerado existen marcados contrastes sociales entre la capital y las ciudades del litoral con respecto al interior del país y las zonas rurales (Míguez, 1999); contrastes que también son grandes dentro de la propia ciudad de Buenos Aires.

En este contexto, la profesión médica se desarrolla como un actor a la vez homogéneo y múltiple: los médicos comparten una serie de preocupaciones vinculadas a la "cuestión social", a la percepción de su papel como reformadores sociales y una "cultura científica" (Terán, 2000), pero lo expresan desde diferentes perspectivas ideológicas, que van desde el liberalismo y el catolicismo social hasta el socialismo o las propuestas higiénico-naturistas de algunos anarquistas (Belmartino, 2005; Lobato, 1996). La mayor parte de ellos pueden ser situados como parte de la "cultura liberal reformista" que prospera entre políticos e intelectuales argentinos entre fines del siglo XIX y las primeras décadas del XX (Zimmermann, 1994).

Entre los problemas vinculados a la cuestión social en la época estudiada, la mortalidad infantil ocupa un lugar central. Se considera que la producción de futuros ciudadanos se ve especialmente amenazada por una elevada mortalidad que afecta principalmente a los recién nacidos y lactantes. ${ }^{2}$ Para explicarla, los argumentos que remiten a las condiciones de vida de los sectores pobres se combinan con aquellos que aluden a la "ignorancia" de las mujeres madres de diferentes sectores sociales. De modo semejante a lo estudiado por Armstrong (1986) para el caso de Gran Bretaña, la tasa de mortalidad infantil es construida como uno de los principales indicadores del "estado material y moral de una nación" (Carreño, Schwarcz, 1927, p.89), enmarcada en la preocupación demográfica por el lento crecimiento vegetativo de la población y unida a una inquietud eugenésica acerca de la calidad de esa población: su "composición racial, social y moral" (p.89). ${ }^{3}$

El combate a la mortalidad infantil, ligado al lugar particular que pasa a ocupar la infancia, se produce de conjunto con los comienzos de la constitución de la pediatría como especialidad médica. Así, en el marco local, pero también formando parte de un movimiento existente a nivel internacional. A partir de la última década del siglo XIX la pediatría comienza a delinearse dentro de las ciencias médicas como un saber especializado en el niño. ${ }^{4}$ Siguiendo especialmente las escuelas pediátricas francesa y alemana, este proceso se produce más o menos simultáneamente en los diferentes países de América Latina (Puga, 2007; Birn, 2006; Del Castillo Troncoso, 2001; Pereira, 2006; Rodríguez Jiménez, 2007). Los médicos latinoamericanos viajan con frecuencia a Francia y Alemania para visitar y formarse en los servicios de atención de niños de sus hospitales, además de estudiar con literatura médica de esos países. Hacia fines de la década de 1920, como parte de la circulación del conocimiento científico de la época, se suma la referencia a médicos de los Estados Unidos (Birn, 2006).

La necesidad de disminuir la mortalidad infantil y las enfermedades que la provocan no es la única preocupación que en la época suscita la niñez. En Buenos Aires, el abandono, la mendicidad, el trabajo callejero y el delito infantiles van siendo recortados, definidos, explicados como "problemas" en el marco de una serie de representaciones socialmente construidas sobre las características de la infancia; representaciones mediante las cuales se establece el modo en que los sujetos deben atravesar esta etapa y se definen sus 
comportamientos y características físicas "normales". Esos otros "problemas" y categorías ligados a la infancia configuran el contexto más amplio de discusiones en los que las teorías e intervenciones médicas sobre los niños tienen lugar y con las cuales dialogan. En efecto, a partir de fines del siglo XIX, en el marco de la ampliación de la presencia estatal en la asistencia social, una serie de agentes sociales - juristas, pedagogos, filántropos, médicos, entre otros - comienzan a elaborar, compartir y/o disputar nominaciones, clasificaciones y acciones de asistencia y protección, de educación, de castigo, entre otras, sobre la infancia. De allí que se considere necesario situar la constitución de la pediatría como parte de un "campo de la infancia" en proceso de formación en el que participan distintas disciplinas científicas y agentes. ${ }^{5}$ En este proceso, tal como sugieren Plotkin y Zimmermann (2012) o de L'Estoile et al. (2000) para otros contextos, los discursos expertos y las prácticas de intervención estatal se despliegan en un espacio que es en parte común, existiendo entre ambos constantes pasajes y mutuas legitimaciones.

\section{La construcción de la pediatría como especialidad médica}

Para analizar el proceso de especialización de la pediatría, en este trabajo se combinan contribuciones de la perspectiva desarrollada por Bonet (2003) en su análisis de la constitución de la identidad profesional de los médicos de familia con algunos elementos señalados por González Leandri $(1996,2006)$ en su estudio de los comienzos de la profesión médica en Argentina. Para el primero, hablar de la construcción de una especialidad profesional implica dar cuenta de un proceso que involucra dos aspectos simultáneos: la construcción epistemológica de un principio de clasificación - a partir del cual se delimitan nuevos objetos de estudio y/o enfoques - y la institucionalización de un grupo profesional. El segundo, si bien no analiza la conformación de especialidades al interior de la medicina, aporta el énfasis en los procesos de legitimación e institucionalización que se producen tanto al interior del grupo profesional como en su relación con el Estado.

El principio de clasificación que pone en juego la pediatría se funda en la delimitación de etapas del ciclo vital, correspondiendo a un modelo de segmentación de las especialidades médicas que Bonet (2003, p.97) llama "segmentación por edad y sexo". A partir del criterio etario, la primera etapa del ciclo vital es recortada como un momento particular, atribuyendo a los sujetos que lo transitan características singulares que, de acuerdo con los promotores de la especialidad, impedirían su abordaje desde las teorías y prácticas médicas destinadas a otras edades.

A su vez, para que el saber del grupo profesional sobre el objeto de estudio e intervención así recortado sea reconocido en términos de una nueva especialidad, debe obtener algún grado de legitimidad, tanto al interior del campo científico profesional de la medicina, como afuera y más allá del mismo. Ello implica la puesta en juego de diferentes "estrategias de persuasión", orientadas hacia "el adentro" y hacia "el afuera" de la profesión (González Leandri, 1996, p.24). Al interior de las ciencias médicas, dichas estrategias se centran en la demostración de la especificidad anatómica y fisiológica de la niñez y en la institucionalización de los criterios de diferenciación de la nueva especialidad a través de la creación de espacios académicos, eventos científicos, asociaciones profesionales y revistas 
especializadas. Hacia el exterior del campo científico, la legitimación de la pediatría se busca a través de una batalla contra los saberes legos, desestimados en términos de "prejuicios" e "ignorancia", así como en el reconocimiento del Estado y la incidencia en las políticas públicas, pues la profesionalización no resulta posible sin la conformación de una creencia de la población en la eficacia de la medicina y sin su participación como usuaria de los servicios médicos recientemente creados.

\section{El estudio de "un sujeto completamente nuevo"}

El discurso de los comienzos de la pediatría muestra un pasaje del estudio de las manifestaciones de las enfermedades "en" los niños, al de las enfermedades "de" los niños (James et al., 1998). Como se verá, entre las razones esgrimidas por algunos médicos que promueven el proceso de especialización, ya no se trata de la presentación de patologías generales en un cuerpo más pequeño, sino de los modos de enfermar y sanar propios de un sujeto cualitativamente diferente de los adultos. Ello da cuenta del triunfo de una serie de argumentos fundados en el estatuto social específico de la infancia con respecto a aquellos que defienden el carácter común del cuerpo de niños y adultos y, por lo tanto, una atención clínica común a pacientes de cualquier edad (Rivorêdo, 1998). ${ }^{6}$ Ahora bien, ¿cuál es el fundamento atribuido a esa distinción?

Tanto en sus obras eruditas como en las de divulgación, los médicos de la época analizada otorgan a la infancia características fisiológicas, anatómicas y del comportamiento que la distinguen de las restantes etapas de la vida. Tal como lo explica a sus alumnos Luis Morquio (profesor de clínica infantil de la Facultad de Montevideo, asiduo partícipe de los debates entre los médicos de Buenos Aires), ${ }^{7}$ ello hace que aun patologías denominadas de la misma manera que las de los adultos, adopten en el niño manifestaciones y modalidades de evolución diferentes, requiriendo un diagnóstico y una terapéutica especiales:

[En la clínica de niños, a diferencia de las otras materias] El sujeto es completamente nuevo, y si en algunos momentos la misma denominación patológica pudo halagar vuestros oídos, recordando algo conocido, en presencia del hecho clínico habéis encontrado una cosa muy distinta, por su semiología, por sus reacciones especiales, por la modalidad de su evolución y por su terapéutica (Morquio, 1906b, p.398).

Desde esta perspectiva, el niño es visto como "el sujeto más susceptible" al contagio de enfermedades y a los efectos de las variaciones estacionales en su organismo. En el cuerpo infantil, a diferencia del adulto, las enfermedades parecen manifestarse con especial nitidez e intensidad y evolucionan rápidamente hacia un desenlace favorable o desfavorable: "La modalidad característica de la patología infantil son las reacciones intensas y rápidas; la precipitación de los hechos, agravando rápidamente una situación que toma fácilmente el aspecto alarmante; la necesidad, a cada instante, de la medicación de urgencia; el desenlace rápido, en un sentido o en otro" (Morquio, 1906a, p.464).

Al mismo tiempo, en la infancia la prevención muestra mayor eficacia y las enfermedades resultan más evitables. En edades tempranas - se señala - las manifestaciones patológicas varían ampliamente según las características individuales, debido a una presencia más 
"pura" de los factores hereditarios, aún no demasiado moldeados o afectados por el ambiente. Esta concepción del niño como una suerte de "página en blanco", en la que distintas influencias ambientales pueden inscribirse, está en la base de las propuestas eugenésicas preventivas de la época que hacen de la incorporación de elementos de un ambiente adecuado el modo de incidir en la calidad racial de las poblaciones. El entender el cuerpo infantil como un "organismo nuevo", dotado de defensas naturales y de "una tendencia espontánea y natural a reaccionar favorablemente" (Morquio, 1906a, p.465) otorga expectativas favorables acerca de la eficacia de la intervención médica.

En síntesis, tanto en el modo de adquirir y manifestar la enfermedad, como en las posibilidades de cura, el cuerpo del niño es considerado más susceptible, más vulnerable que el de los adultos pero, a la vez, con mayor fortaleza y posibilidades de resistencia y curación. ¿Cómo se explica esta aparente contradicción?

Es la noción de plasticidad, de maleabilidad, atribuida a la infancia - una "blanda masa a ser moldeada" (Aráoz Alfaro, 1929, p.IX) - que implica tanto incompletud como potencialidad, la que da sentido y coherencia a las representaciones que guían la teoría y la práctica de la pediatría: porque el niño aún no ha tomado su forma definitiva es la razón por la que puede reaccionar de una u otra manera ante las enfermedades y la terapéutica. $Y$ este carácter a la vez incompleto y potencial, atribuido a la niñez, se funda en su definición a partir de los procesos de crecimiento y desarrollo.

Crecimiento y desarrollo, entendidos como "las dos funciones esenciales de la vida de un niño" (Morquio, 1906b, p.398), hacen de la infancia una primera etapa vital y del niño un ser inacabado, en proceso de constituirse como un ser humano completo - homologado al adulto - de manera gradual y progresiva. Desde esta concepción, todo estudio o intervención sobre el niño procurará situarlo en un momento o etapa de un recorrido temporal longitudinal.

La apelación a esos procesos evolutivos fundamenta la intervención de los expertos, tanto sobre los aspectos físicos como los "morales", estableciéndose una articulación entre la mirada médica y el tratamiento social del niño, expresado en políticas, leyes o instituciones destinados a la niñez:

Toda la vida del niño está sujeta a su crecimiento y a su desarrollo; esta evolución se opera dentro de reglas fisiológicas que deben ser vigiladas y dirigidas conscientemente, para evitar desviaciones y para corregirlas cuando éstas se produzcan. Es del mayor conocimiento de su naturaleza, de sus condiciones físicas y psíquicas, de las causas que provocan las enfermedades, físico y morales, de la manera de corregirlas, que ha surgido todo un conjunto de problemas, higiénicos y sociales, que se traducen en leyes y organizaciones, que constituyen hoy la defensa del niño en todas sus manifestaciones (Crónica, 1919, p.276).

Crecimiento y desarrollo se constituyen así en la base de una distinción de carácter evolutivo entre niñez y adultez; distinción que persiste en la teoría pediátrica hasta la actualidad. A partir de centrar la definición de la niñez en su evolución, la pediatría no solo busca dedicarse al estudio y tratamiento de las enfermedades infantiles, sino también, al seguimiento de dichos procesos en el niño sano, a fin de prevenir posibles desviaciones del recorrido esperable. 


\section{Procesos académicos y asociativos}

En consonancia con la delimitación del niño como objeto de estudio y tratamiento, la pediatría se institucionaliza como rama o especialidad médica y los pediatras comienzan a constituirse en un grupo profesional. Ello se da a través de la búsqueda de diferenciación y reconocimiento al interior de las ciencias médicas, mediante procesos académicos y asociativos. Al mismo tiempo, el lugar central que ocupa la infancia en los problemas sociales construidos entre fines del siglo XIX y comienzos del XX, permite a esos profesionales atribuirse el ejercicio de una "misión social" y reclamar un lugar específico en el campo social más amplio, situándose como representantes de un saber experto requerido por el Estado.

En el espacio académico, las transformaciones de la carrera de medicina de la Universidad de Buenos Aires muestran que en 1883 es nombrado el primer profesor de la "cátedra de enfermedades de los niños y clínica respectiva", Manuel Blancas, ${ }^{8}$ pasando la medicina de la niñez a ser tratada en una materia específica. Hasta entonces, los contenidos referidos a las enfermedades en la infancia habían estado incluidos en los programas de las cátedras de clínica médica o de partos (Puga, 2007). A partir de 1906 la cátedra se denomina de "clínica pediátrica" y, en 1907, Ángel Centeno reemplaza a Blancas como profesor titular. ${ }^{9}$ En 1919, en coincidencia con el nombramiento de Mamerto Acuña ${ }^{10}$ como nuevo profesor, la cátedra pasa a llamarse "clínica de pediatría y puericultura", incorporando explícitamente el aspecto preventivo e higiénico al abordaje clínico. En el proceso académico de conformación del perfil profesional del pediatra se percibe, en la primera década del siglo XX, una tensión entre el modelo de la clínica clásica, fundada en la observación directa, la percepción aguzada, la capacidad de interpretar los síntomas y un modelo considerado más moderno que basa los diagnósticos en la experimentación, la incipiente radiología y los análisis de laboratorio (Centeno, 1907).

Paralelamente, comienzan a crearse servicios de atención de enfermedades de los niños en hospitales generales y en 1908 se construye una nueva sede para el Hospital de Niños de Buenos Aires, permitiendo ampliar y complejizar su atención como Hospital Docente. ${ }^{11}$ En 1905, como parte de esas transformaciones institucionales, la Casa Cuna de Buenos Aires, perteneciente a la Sociedad de Beneficencia, es transformada en Hospital de Niños Expósitos. Cabe aclarar que la pediatría se constituye en una época en que el Estado avanza en la creación, organización y supervisión de un sistema de atención de la salud, pero instituciones filantrópicas como la Sociedad de Beneficencia aún poseen y administran gran parte de los establecimientos destinados a tal fin. ${ }^{12} \mathrm{Al}$ interior de los mismos, son frecuentes las tensiones acerca de los criterios de atención de la salud entre las damas que administran la Sociedad y los médicos que integran los cuerpos consultivos y asesores (Pita, 2012). En este marco, los médicos de niños participan de una disputa más amplia entre los defensores de una administración de la asistencia médica y social totalmente estatal, y aquellos que proponen una combinación de supervisión estatal e iniciativa privada, tal como lo expone Nari (2004).

El proceso de especialización también se visualiza en la creación de publicaciones y de eventos científicos dedicados exclusivamente al tratamiento médico de la niñez. 
La Semana Médica, publicación semanal de la Academia de Medicina, incluye, desde su comienzo en 1894, artículos sobre enfermedades presentes en los niños ("Convulsiones en la infancia", "Diarrea en los niños"), así como una preocupación por la mortalidad infantil a través de estadísticas presentadas en informes periódicos. En 1900 - mostrando el pasaje al estudio de las "de" los niños - pasa a tener una sección denominada "Pediatría", con artículos generales sobre tratamiento del niño, y otra llamada "Clínica Pediátrica", centrada en casos clínicos.

Durante la década de 1890 se fundan la Revista de Higiene Infantil, del Patronato de la Infancia y la Revista del Hospital de Niños Ricardo Gutiérrez. En 1905, con la creación de los Archivos Latinoamericanos de Pediatría: Revista Mensual Internacional, el nuevo colectivo profesional propone contar con un órgano de comunicación y discusión científica propio, de alcance latinoamericano, que responda a la especificidad de la pediatría dentro de la medicina:

Ilustrados colegas:

La especialización en el dominio de la medicina infantil está ya demasiado extendida en toda América Latina para que los médicos de niños sigamos publicando el fruto de nuestra observación y de nuestros trabajos en las revistas generales de medicina.

Como en Europa, los médicos que en estos países nos ocupamos de higiene y de medicina infantil, necesitamos de órganos especiales, y hemos creído por eso de positivo interés general reunir toda la producción pediátrica latinoamericana en una sola revista, que constituya así una verdadera 'hoja central' tan completa como sea posible, no sólo para la medicina y la cirugía, sino también para la higiene de la infancia (Editorial, 1905, p.2).

Los Archivos se publican mensualmente, incluyendo artículos en castellano, portugués y francés. El comité editorial está integrado por algunos de los médicos con mayor reconocimiento académico y estatal de Brasil, Uruguay y Argentina: Olinto de Oliveira (Porto Alegre), Fernandes Figueira (Rio de Janeiro), Luis Morquio (Montevideo) y Gregorio Aráoz Alfaro (Buenos Aires). Algunos de ellos han participado en la redacción de la segunda edición del Traité des maladies des enfants, de los médicos franceses Granchet y Comby, texto de referencia en la formación de los médicos argentinos, publicado en 1905.

La revista presenta diversas secciones temáticas: "Observaciones clínicas", "Artículos originales", "Protección y asistencia de la infancia", "Análisis bibliográficos", "Cuestiones del día", "Crónica". Esta última incluye los relatos de visitas científicas a los más prestigiosos servicios de pediatría de Francia, Alemania o, ${ }^{13}$ más tarde, de los EEUU, así como comentarios sobre la participación en congresos internacionales, permitiendo percibir la intensa circulación internacional de ideas científicas que se produce en la época y que, como señala Birn (2006), está lejos de ser unidireccional, implicando un activo intercambio y una adaptación selectiva al propio contexto de las teorías científicas y políticas sociales del exterior. Si bien participan de la preocupación internacionalmente compartida acerca de la mortalidad infantil, los médicos de Buenos Aires la analizan en relación con el déficit poblacional de un Estado recientemente organizado y procuran sopesar la posible eficacia de políticas e instituciones sanitarias europeas en una "joven nación" cuya población es vista como racialmente heterogénea. Es necesario aclarar que, lejos de tener un alcance nacional, ese intercambio se restringe principalmente a los médicos de Buenos Aires y 
algunos de Rosario, quienes configuran canales de comunicación que los vinculan más estrechamente con colegas de París, de Montevideo y de algunos centros urbanos de Brasil, que con los médicos del interior del país.

La publicación de los Archivos se interrumpe entre 1909 y 1911, cuando la revista comienza a ser bimestral y los médicos brasileños dejan de participar en su dirección. La redacción pasa a organizarse en tres secciones principales, cada una con su secretario: medicina, cirugía, higiene y protección de la infancia. La revista es reemplazada por los Archivos Argentinos de Pediatría en 1929, cuando cada uno de los países que la integraba originalmente cuenta con una abundante producción científica y un desarrollo institucional propios.

En el proyecto editorial de la revista puede verse no solo la búsqueda de participación en el debate científico internacional sino también un modo de afirmación del reciente colectivo profesional y de sus propuestas asociativistas. Es a través de los Archivos que se difunden las convocatorias para la conformación de las primeras asociaciones profesionales de pediatras a nivel nacional e internacional, así como para la realización de los primeros congresos científicos de la nueva especialidad. Así, en el número de abril de 1907 circula una primera invitación en francés a conformar la Société Internationale de Pédiatrie, enviada por un médico de la Universidad de Járkov, Ucrania. Esta propuesta busca explícitamente el reconocimiento y legitimación, al interior de las ciencias médicas, de la pediatría como una rama independiente y de similar importancia a la de la clínica general, apelando al plus que le otorga su función social de cuidado temprano de las poblaciones: "una sociedad internacional - se afirma - es indispensable para otorgar a la pediatría el lugar que le pertenece por derecho y para recompensarla así por todos los beneficios que ella ya ha brindado a la humanidad" (Sociétés..., 1907, p.138). ${ }^{14}$

Siguiendo esta tendencia internacional, por iniciativa del reconocido médico Aráoz Alfaro, en octubre de 1911, se funda la Sociedad Argentina de Pediatría que hace de los Archivos su órgano oficial. Dicha fundación se produce en una reunión de médicos que se autodenominan pediatras, realizada en la casa de Aráoz Alfaro. Allí se nombra una comisión compuesta por éste, Genaro Sisto y Ernesto Gaing, para redactar los Estatutos y Reglamentos de la nueva Sociedad. Estos son posteriormente discutidos en una asamblea realizada en la Sociedad Médica Argentina, como modo de obtener legitimidad institucional, constituyéndose efectivamente la Sociedad con la elección por voto secreto de su comisión directiva, conformada por Centeno (presidente), Aráoz Alfaro (vicepresidente), Sisto (secretario general), Gaing (secretario de actas), Acuña (bibliotecario), Arraga, Castro y Viñas (vocales). La nueva asociación queda liderada en gran parte por el núcleo que había colaborado en la edición de 1905 del Tratado de Granchet y Colby, proceso que reforzó su prestigio científico y académico.

La trayectoria de los fundadores combina la actividad académica y asociativa con otras ligadas a la función pública en instituciones dependientes de la asistencia pública municipal, el asesoramiento en instituciones de beneficencia, la atención de pacientes en consultorios particulares y la escritura de textos de divulgación. Un caso especialmente claro es el de Aráoz Alfaro (1870-1955), quien a lo largo de sus vida profesional ejerce como profesor universitario, jefe del Servicio de Niños del Hospital San Roque, presidente de la Academia 
Nacional de Medicina, secretario de la Asistencia Pública, fundador y primer vicepresidente de la Sociedad Argentina de Pediatría, director de los Archivos Latinoamericanos de Pediatría, presidente del Departamento Nacional de Higiene, miembro correspondiente de sociedades médicas extranjeras y autor de numerosos textos de divulgación. Cabe pensar que no se trata solo de una combinación de diferentes tareas y espacios de ejercicio profesional, sino de una conjugación de ámbitos que de alguna manera potencia la legitimación del abordaje pediátrico de la infancia: no es solo apelando a la especificidad del cuerpo infantil como objeto de estudio e intervención como se fundamenta la delimitación de la nueva especialidad, sino también invocando el carácter de "misión social" de su lucha contra la mortalidad infantil, realizada mediante la participación de los médicos de niños en espacios de gestión de la salud pública.

Una de las tareas centrales para el cumplimiento de la finalidad científica que se atribuyen las nuevas asociaciones de pediatras es la organización de congresos de la especialidad, como el primer Congreso de la Asociación Internacional de Pediatría, realizado en 1912, en París, con la presencia de varios médicos latinoamericanos o la participación en congresos más amplios, centrados en la asistencia a la infancia, como los congresos internacionales Des Gouttes de Lait ("De las Gotas de Leche"). Los pediatras argentinos ocupan un lugar destacado en la organización del primer Congreso Americano del Niño, realizado en Buenos Aires, en 1913, y participan activamente en el segundo, realizado en Montevideo, en 1919.15

Sin embargo, esta creciente división del trabajo y especialización al interior de la medicina, con la consiguiente tendencia a la construcción de monopolios profesionales, no se traduce en la época en incumbencias excluyentes, estando la profesión médica coordinada "por redes informales de referencia entre generalistas y especialistas, por relaciones flexibles de equipo médico o entre médicos y hospitales" (Belmartino, 2005, p.24). Tampoco puede ser entendida como un proceso generalizado, restringiéndose el proceso aquí estudiado más bien a círculos académicos, científicos y algunas instituciones sanitarias, estatales de grandes centros urbanos como Buenos Aires. En ese marco, la conformación de una especialidad médica orientada a la niñez no implica una exclusividad de los pediatras en el tratamiento médico de los niños quienes, en gran parte, y sobre todo en zonas rurales y ciudades pequeñas, seguirán siendo atendidos por los médicos clínicos generales. No obstante, son los pediatras que participan en los espacios académicos y de atención en los servicios especializados de los hospitales quienes, cada vez más, generan y validan el saber médico sobre el niño, transmitiéndolo a los médicos sin especialización.

\section{Reconocimiento estatal y disputas con los saberes legos}

Como fuera mencionado, la constitución de la pediatría como especialidad médica requiere también la puesta en juego de "estrategias de persuasión" (González Leandri, 1996) orientadas a las agencias estatales y a la población en general que le permitan construir su legitimidad más allá del campo profesional. La producción de saberes en los espacios académicos y asociativos analizados se da de conjunto con la participación de los primeros pediatras en los espacios estatales de atención de la salud, en una relación ambigua y cambiante en la que los grupos profesionales proporcionan sustento científico 
y responden a las demandas del Estado, a la vez que exigen y promueven reformas en sus instituciones, construyendo su legitimidad como expertos (Biernat, Ramacciotti, 2013).

En ese proceso resultan claves el lugar social de la infancia y la centralidad de los problemas construidos en torno de ella, vinculados con una nueva sensibilidad que la concibe en términos de fragilidad, maleabilidad, potencialidad, y la anuda a una noción de maternidad naturalizada y sacralizada. El presentar su quehacer en términos de la "misión social" del tratamiento de la infancia vulnerable, pero desde el lugar de un saber experto, fundado en la ciencia, permite a los médicos de niños participar activamente en organismos estatales y en el diseño de políticas públicas y, desde allí, emprender una constante e inacabada disputa con otros saberes y prácticas (populares, tradicionales, religiosos) de atención infantil. Es justamente este carácter multifacético el que, en una época donde las especialidades médicas no están rígidamente delimitadas, habilita a estos profesionales a reclamar un lugar específico tanto en el campo académico-científico como en el campo social más amplio.

Además de los servicios de atención infantil en los hospitales de la ciudad, entre los establecimientos cuya creación promueven la asistencia a los niños pueden mencionarse las "gotas de leche"16 y aquellos fundados a partir de la creación, en 1908, de la Dirección de Primera Infancia de la Asistencia Pública de Buenos Aires: dispensarios de lactantes, institutos de puericultura y oficinas de inspección de nodrizas. ${ }^{17}$ Concebidos como ámbitos al mismo tiempo asistenciales y educativos, centran su abordaje en la puericultura, entendida como el área de la medicina del niño especializada en la crianza infantil, especialmente durante la "primera infancia". ${ }^{18}$

La puericultura se constituye como parte de la pediatría, aunque con una relativa autonomía con respecto a ella. ${ }^{19}$ A diferencia de la clínica pediátrica, se orienta hacia los cuidados cotidianos del niño sano mediante la transmisión a las madres de métodos de crianza considerados racionales y científicos, procurando que la guía de la ciencia médica tome en la crianza del niño el lugar hasta entonces ocupado por los consejos de los curadores populares y de las mujeres de la familia. Estos saberes, fuentes habituales de conocimiento acerca de la crianza infantil, pasan a ser fuertemente desautorizados por los pediatras en términos de "ignorancia" y "prejuicios". La estrategia de intervención y persuasión puesta en juego para combatirlos es de carácter pedagógico y preventivo. Para ello, se emprenden diferentes acciones, que van desde la publicación de cartillas, folletos y manuales de crianza infantil, hasta las visitas domiciliarias y el seguimiento periódico del niño en los establecimientos de la Dirección de la Primera Infancia. Estas acciones pueden ser consideradas como parte de un trabajo minucioso, nunca del todo concluido, de implantación de una creencia en el valor de la salud infantil y en la capacidad objetiva y racional de la ciencia para asegurarlo (Boltanski, 1969).

Si bien efectuar un balance de los resultados de ese proceso requeriría la realización de otro trabajo, pueden señalarse algunos elementos que surgen de datos estadísticos (Coni, 1920; Nari, 2004), de los informes periódicos que elaboran los médicos encargados de los mencionados establecimientos asistenciales (Foster, 1906, 1907, 1911; Oliva, 1917, 1918), así como de las quejas sobre las prácticas maternas que aquellos reiteran en diferentes escritos. Estos datos permiten inferir que, aunque hay un leve aumento del porcentaje de 
niños atendidos en dispensarios, institutos de puericultura y "gotas de leche", así como un descenso de las cifras de mortalidad infantil en la ciudad de Buenos Aires, la concurrencia de la población a esos establecimientos dista mucho de ser regular y suele ocurrir cuando los niños ya están enfermos. Así, la clientela que los pediatras de los servicios públicos de salud logran atraer parece estar compuesta mayormente por mujeres cuyos hijos padecen problemas de salud o requieren de asistencia alimentaria. Más difícil parece ser lograr que se sometan a la guía del médico las madres de niños sanos, a las que posiblemente su grupo familiar provea de los conocimientos necesarios para la crianza. Así, por lo menos hasta comienzos de la década de 1920, la medicalización sería más intensa con respecto a la enfermedad infantil que al cuidado del niño sano. ${ }^{20}$

\section{Consideraciones finales}

A lo largo del recorrido realizado se ha procurado mostrar algunos elementos que permiten dar cuenta de la constitución de una especialidad médica, nominada en términos de pediatría y centrada en la especificidad atribuida a la infancia, en el contexto de Buenos Aires, entre 1890 y 1920. Recuperar los trazos principales que delinean dicho proceso y que aparecen como tales ante una mirada retrospectiva, no implica atribuirles un carácter de unilinealidad o nitidez, ni tampoco concebirlos como parte de algún tipo de "evolución interna" de la propia medicina, sino intentar comprender la construcción epistemológica e institucional a partir de la cual la pediatría se constituye como un saber experto en un campo de la infancia en proceso de formación.

Ello requiere tener en cuenta el lugar que la niñez ocupa en las preocupaciones sociales de la época, pues la medicina del niño o pediatría y la categoría social de niñez o infancia son producidas - en sus comienzos, pero también actualmente - como parte de un conjunto más amplio de prácticas sociales orientadas a la "conservación de los niños" (Castellanos, 2003) en el marco de la denominada "cuestión social". En este sentido, es posible afirmar que existe una relación mutuamente constitutiva entre la estructuración de una especialización profesional y la producción de una categoría social, pues no ha sido posible concebir una especialidad médica para los niños sin que estos fueran reconocidos con un estatuto social particular pero, al mismo tiempo, la pediatría habría contribuido a definir y naturalizar la categoría y el lugar social de la infancia.

En el mismo movimiento en que construyen su objeto de estudio e intervención a partir de la especificidad atribuida a la niñez y al cuerpo infantil, ciertos médicos desarrollan un conjunto de procesos académicos y asociativos mediante los que se constituyen como grupo profesional y recortan su saber como una especialidad al interior de la medicina. Al mismo tiempo, procuran ser legitimados e incidir en el campo social más amplio, mediante la participación en distintas instancias estatales de gestión y atención de la salud infantil. De allí la necesidad de vincular las prácticas de diferenciación al interior de la ciencia médica y la construcción de un discurso experto sobre la niñez, con las prácticas estatales vinculadas a la política poblacional (L'Estoile et al., 2000). En este proceso, la pediatría se constituye e institucionaliza como una especialidad médica que busca intervenir no solo sobre el cuerpo enfermo del niño, sino sobre sus condiciones saludables - su crecimiento y 
desarrollo - en el marco de las preocupaciones demográficas que acompañaron el proceso de construcción de la nación desde fines del siglo XIX.

Pero, si a comienzos del siglo XX la profesión médica ya ha logrado el reconocimiento jurídico que le otorga derecho exclusivo en la cura de enfermedades y la pediatría se consolida como especialidad, el cuidado cotidiano del niño sano continúa siendo considerado por gran parte de la población como una atribución femenina y propia del ámbito doméstico. Esto hará del ejercicio del saber y de la práctica médica sobre la infancia y la crianza un conflicto constante, aún inacabado, con otros saberes y prácticas; conflicto a través del cual no solo se busca imponer un conjunto de pautas y principios sobre el cuidado infantil, sino un modo de entender la niñez y definir su lugar con respecto a otros grupos sociales.

\section{NOTAS}

${ }^{1}$ El concepto general de "cuestión social" es tomado de los planteos de Castel (1997). Para la caracterización de la "cuestión social" en Argentina en relación al tema aquí abordado, ver Tenti Fanfani (1990) y González Leandri (2000).

${ }^{2}$ El trabajo con las estadísticas en el periodo considerado presenta dificultades, sobre todo para las cifras a nivel nacional. En la ciudad de Buenos Aires, en 1900, las defunciones de niños menores de un año representan el $54,5 \%$ del total de muertes acontecidas y las de niños de 0 a 5 años, el 44,1\% (Nari, 2004). A nivel nacional, según el trabajo de Otero (2007), la mortalidad infantil es en 1910 de 147,8 niños menores de un año muertos cada mil nacidos vivos. En 1914, esta cifra ha descendido a 116 por mil y en la década de 1930, a 97,3 por mil.

${ }^{3}$ En Argentina, las propuestas eugenésicas de los estudios científicos positivistas y los proyectos políticos locales, por lo menos hasta entrada la década de 1920, son de carácter preventivo: orientadas a crear una "raza" nueva a partir de la existente y con influencia de las ideas del "transformismo" neolamarckiano, que sostiene la posibilidad de transformación de los individuos y las "razas" a partir de la incorporación de caracteres del medio. Hacia la década de 1930, las propuestas eugenésicas se endurecen (Nari, 2004).

${ }^{4}$ Badinter (1985) registra que las denominaciones de "pediatría" y "puericultura" que adopta la nueva especialidad aparecen por primera vez en Francia en 1872 y 1864, respectivamente.

${ }^{5}$ El concepto de "campo" es empleado en el sentido que le atribuye Bourdieu (1990).

${ }^{6}$ Rivorêdo (1998) sitúa esta polémica sobre la pertinencia de la atención médica de los niños entre fines del siglo XVIII y comienzos del XIX. Si bien la ubica como un "primer momento de afirmación de la pediatría" (p.38), aquí se considera que se trató más bien de debates que precedieron y generaron ciertas condiciones conceptuales para el efectivo surgimiento de la especialidad a fines del siglo XIX y comienzos del XX.

${ }^{7}$ Si bien el eje de la investigación se situó en las obras de pediatras que trabajaron en Argentina, se decidió incluir en el análisis los textos del uruguayo Morquio (1867-1935) por mantener las universidades de Montevideo y Buenos Aires un intercambio constante de ideas y profesionales y ser aquel una referencia constante para la consulta de los pediatras argentinos. Desde 1894 dirige el Asilo de Expósitos y Huérfanos de Montevideo y en 1900 es nombrado profesor titular de medicina infantil de la Universidad de la República. Para un análisis completo de su trayectoria, ver Birn (2006).

${ }^{8}$ Español, graduado en medicina en Montevideo. Blancas (1823-1906) combina su cargo de profesor en la Universidad de Buenos Aires con la dirección de la Casa Cuna - más tarde transformada en Hospital de Niños Expósitos -, que ejerce desde 1855.

${ }^{9}$ Como su antecesor, Centeno combina su desempeño como profesor titular de clínica pediátrica con el de director del Hospital de Niños Expósitos.

${ }^{10}$ Además de desempeñarse como profesor universitario, dirige el Hospital de Clínicas.

${ }^{11}$ El Hospital de Niños de la Ciudad de Buenos Aires es fundado en 1875 bajo dependencia de la Sociedad de Beneficencia. En 1883 se convierte en Hospital Docente en consonancia con la creación de la cátedra de enfermedades de los niños y clínica respectiva. 
${ }^{12}$ La Sociedad de Beneficencia, creada en 1823 durante el gobierno de Rivadavia para organizar $\mathrm{n}$ gran parte la educación y la asistencia social en general, ha perdido para la época aquí analizada gran parte de su poder e incumbencias iniciales pero continúa dirigiendo varios hospitales de la ciudad de Buenos Aires. Mantuvo siempre una relación ambigua con el Estado; ambigüedad derivada de su carácter de entidad privada creada y financiada mayormente por el Estado para cumplir funciones públicas.

${ }^{13}$ De la pediatría francesa se valoran los aportes del conocimiento clínico y la creación de instituciones de protección de la primera infancia, como las "gotas de leche" y los consultorios de lactantes. Ya entrada la primera década del siglo XX, se resaltan las contribuciones en microbiología y nutrición de la pediatría alemana (De Asúa, 2012).

${ }^{14}$ En ésta y en las demás citas de textos publicados en otros idiomas la traducción es libre.

${ }^{15}$ Nari (2004) señala que los congresos del niño de América del Sur comenzaron siendo impulsados por médicas y militantes feministas de la región quienes, sin embargo, habrían ido perdiendo terreno en relación con los médicos pediatras e higienistas. Para un estudio más detallado sobre los primeros congresos del niño, ver Guy (1998).

${ }^{16}$ Iniciadas en 1904, replicando las existentes en Francia, proporcionan leche. Comienzan como iniciativa privada y luego reciben financiación municipal.

${ }^{17}$ Ubicados en barrios pobres de la ciudad, los dispensarios de lactantes preparan y entregan alimentos y son también consultorios externos para recién nacidos donde se realiza una "asistencia educativa" a las madres. En los institutos de puericultura se hace el seguimiento médico del recién nacido y hay salas de internación. La Oficina de Inspección de Nodrizas controla las condiciones de salubridad de la leche de las mujeres empleadas como de nodrizas, así como la alimentación y cuidado que reciben sus propios hijos. Para un abordaje más detallado de estas instituciones y sus estrategias, ver Colangelo (2012) y Billorou (2008).

${ }^{18}$ Este desarrollo institucional destinado a la niñez no puede generalizarse para todo el territorio argentino, pues al interior del país, recién entrada la década de 1930, se le destinan políticas nacionales centralizadas en el Departamento Nacional de Higiene (Billorou, 2008).

${ }^{19}$ La delimitación disciplinaria y la relación entre ambas no están definidas de manera clara ni uniforme en la época estudiada. Para algunos autores de la época, la puericultura es una parte de la pediatría; para otros, ambos abordajes de la niñez constituyen disciplinas con igual rango dentro de la medicina, haciendo imposible proyectar hacia la época estudiada los actuales criterios de distinción entre especialidades.

${ }^{20}$ Para un análisis más detallado de las transformaciones y resistencias producidas ante la medicalización del cuidado infantil, ver Colangelo (2012).

\section{REFERENCIAS}

ÁlVAREZ, Adriana; CARBONETTI, Adrián (Comp.).

Saberes y prácticas médicas en la Argentina. Mar del Plata: Eudem. 2008.

ARÁOZ ALFARO, Gregorio.

El libro de las madres: manual práctico de higiene del niño, con indicaciones sobre el embarazo, parto y tratamiento de accidentes. Buenos Aires: Cabaut. 1929.

ARIÈS, Philippe.

História social da criança e da família. Rio de Janeiro: Guanabara Koogan. 1981.

ARMSTRONG, David.

The invention of infant mortality. Sociology of Health and Illness, v.8, n.3, p.211-232. 1986.

ARMUS, Diego.

¿Qué historia de la salud y la enfermedad? Salud

Colectiva, v.6, n.1, p.5-10. 2010.
ARMUS, Diego (Comp.).

Avatares de la medicalización en América Latina, 1870-1970. Buenos Aires: Lugar Editorial. 2005.

ARMUS, Diego; BELMARTINO, Susana.

Enfermedades, médicos y cultura higiénica. In: Cattaruzza, Alejandro (Dir.). Nueva Historia Argentina. t.7. Buenos Aires: Sudamericana. p.283-329. 2001.

BADINTER, Elisabeth.

Um amor conquistado: o mito do amor materno. Rio de Janeiro: Nova Fronteira. 1985.

BELMARTINO, Susana.

La atención médica argentina en el siglo XX: instituciones y procesos. Buenos Aires: Siglo XXI. 2005.

BIERNAT, Carolina; RAMACCIOTTI, Karina. Crecer y multiplicarse: la política sanitaria materno-infantil argentina, 1900-1960. Buenos Aires: Biblos. 2013. 
BIERNAT, Carolina; RAMACCIOTTI, Karina. La tutela estatal de la madre y el niño: estructuras administrativas, legislación y cuadros técnicos, 1936-1955. História, Ciências, Saúde-Manguinhos, v.15, n.2, p.331-351. 2008.

BILLOROU, María José.

La protección maternal e infantil debe ser colocada en primer plano en un país como el nuestro: las políticas de protección a la infancia en la Argentina de principios del siglo XX. Trabajo presentado en la Jornada "Historia de la Infancia en Argentina, 1880-1960". Los Polvorines: Universidad Nacional de General Sarmiento. 2008.

BIRN, Anne-Emanuelle.

O nexo nacional-internacional na saúde pública: o Uruguai e a circulação das políticas e ideologias de saúde infantil, 1890-1940. História, Ciências, Saúde-Manguinhos, v.13, n.3, p.675-708. 2006.

BOLTANSKI, Luc.

Prime éducation et morale de classe. Paris: Mouton. 1969.

BONET, Octavio.

Os médicos da pessoa: um estudo comparativo sobre a construção de uma identidade profissional. Tese (Doutorado em Antropologia Social) - Universidade Federal do Rio de Janeiro, Rio de Janeiro. 2003.

BOURDIEU, Pierre.

Sociología y cultura. México: Grijalbo. 1990.

BRIOLOTTI, Ana.

Educando a los padres argentinos: un análisis a través de los manuales de puericultura de Aráoz Alfaro y Garrahan. Avances del Cesor, v.13, n.15, p.39-60. 2016.

CARREÑO, Carlos; SCHWARCZ, Ricardo.

El niño (su higiene, sus cuidados). Buenos Aires: L. Gutiérrez. 1927.

CASTEL, Robert.

La metamorfosis de la cuestión social: una crónica del salariado. Buenos Aires: Paidós. 1997.

CASTELLANOS, Marcelo E.P.

A pediatria e a construção social da infância: uma análise do discurso médico-pediátrico. Dissertação (Mestrado em Saúde Coletiva) - Universidade Estadual de Campinas, Campinas. 2003.

CENTENO, Ángel.

Lección inaugural del curso de 1907. Archivos Latinoamericanos de Pediatría, año 3, n.4, p.113115. 1907.

COLANGELO, María Adelaida.

La crianza en disputa: medicalización del cuidado infantil en la Argentina entre 1890 y 1930. Tese (Doctorado en Ciencias Naturales - opción Antropología) - Universidad Nacional de La Plata, La Plata. 2012.

CONI, Emilio.

Demografía nacional: mortalidad infantil en

la Argentina, durante el sexenio 1911-1916.

Archivos Latinoamericanos de Pediatría, t.14, n.6, p.496-499. 1920.

COSSE, Isabella et al.

Infancias, políticas y saberes en Argentina y Brasil, siglos XIX y XX. Buenos Aires: Teseo. 2011.

COWEN, Miguel P.

Nacimientos, partos y problemas de la primera infancia: fines del siglo XVIII, primeras décadas del siglo XIX. In: Moreno, José Luis (Ed.). La política social antes de la política social. Buenos Aires: Prometeo. p.47-90. 2000.

CRÓNICA.

Crónica. Archivos Latinoamericanos de Pediatría, t.13, n.1, p.274-283. 1919.

DE ASÚA, Miguel.

La pediatría como disciplina cultural y social. Archivos Argentinos de Pediatría, v.110, n.3, p.231236. 2012.

DEL CASTILLO TRONCOSO, Alberto.

Moral médica y secularización: el cuerpo infantil en el discurso médico del porfiriato. Política y Cultura, n.16, p.1-23. 2001.

DI LISCIA, María Silvia.

Dentro y fuera del hogar: mujeres, familias y medicalización en Argentina, 1870-1940. Signos Históricos, n.13, p.94-119. 2005.

DUARTE, Luis Fernando Dias.

Prefácio. In: Rohden, Fabíola. Uma ciência da diferença: sexo e gênero na medicina da mulher. Rio de Janeiro: Editora Fiocruz. p.7-9. 2001.

EDITORIAL.

Editorial. Archivos Latinoamericanos de Pediatría, año 1, n.1, p.2-3. 1905.

FOSTER, Enrique.

Protección y asistencia de la infancia: memoria presentada a la dirección de la Asistencia Pública y correspondiente al año 1910. Archivos Latinoamericanos de Pediatría, año 1, t.5, n.1, p.122-139. 1911.

FOSTER, Enrique.

Memoria sobre el funcionamiento del dispensario de lactantes "La Gota de Leche". Archivos Latinoamericanos de Pediatría, año 3, n.3, p.104-110. 1907.

FOSTER, Enrique.

Memoria sobre el funcionamiento de la "Gota de Leche". Archivos Latinoamericanos de Pediatría, año 2, n.7, p.275-281. 1906. 
GONZÁLEZ LEANDRI, Ricardo.

Itinerarios de la profesión médica y sus saberes de Estado. Buenos Aires, 1850-1910. In: Plotkin, Mariano B.; Zimmermann, Eduardo. Los saberes del Estado. Buenos Aires: Edhasa. p.125-152. 2012.

GONZÁLEZ LEANDRI, Ricardo.

La consolidación de una inteligentzia médico profesional en Argentina, 1880-19000. Diálogos: Revista Electrónica de Historia, v.7, n.1, p.37-78. 2006.

GONZÁLEZ LEANDRI, Ricardo.

Miradas médicas sobre la cuestión social. Buenos Aires a fines del siglo XIX y principios del XX. Revista de Indias, v.60, n.219, p.421-435. 2000.

GONZÁLEZ LEANDRI, Ricardo.

La profesión médica en Buenos Aires, 18521870. In: Lobato, Mirta. Política, médicos y enfermedades: lecturas de historia de la salud argentina. Buenos Aires: Biblos. p.21-53. 1996.

GUY, Donna.

The Pan American Child Congresses, 1916-1942: Pan Americanism, child reform and welfare state in Latin America. Journal of Family History, v.23, n.3, p.272-291. 1998.

JAMES, Allison et al.

Theorizing childhood. Cambridge: Polity Press. 1998.

L'ESTOILE, Benoît et al.

Savoirs anthropologiques, administration des populations et constructions de l'État. Revue de Synthèse, v.121, n.3-4, p.233-264. 2000.

LIONETTI, Lucía; MíGUEZ, Miguel (Comp.). Las infancias en la historia argentina: intersecciones entre prácticas, discursos e instituciones, 18901960. Rosario: Prohistoria. 2010.

LOBATO, Mirta (Ed.).

Política, médicos y enfermedades: lecturas de historia de la salud en Argentina. Buenos Aires: Biblos; Universidad Nacional de Mar del Plata. 1996.

MíGUEZ, Eduardo J.

Familias de clase media: la formación de un modelo. In: Devoto, Fernando; Madero, Marta. Historia de la vida privada en Argentina. t.2. Buenos Aires: Taurus. p.21-45. 1999.

MORQUIO, Luis.

La pediatría moderna en sus relaciones con la vida profesional (lección de fin de curso, segunda parte). Archivos Latinoamericanos de Pediatría, año 2, n.12, p.444-465. 1906a.

MORQUIO, Luis.

La pediatría moderna en sus relaciones con la vida profesional (lección de fin de curso). Archivos Latinoamericanos de Pediatría, año 2, n.11, p.397-416. 1906b.
NARI, Marcela.

Políticas de maternidad y maternalismo político. Buenos Aires: Biblos. 2004.

OLIVA, Silvestre.

Protección de la primera infancia: memoria y estadística correspondiente al año 1917. Archivos Latinoamericanos de Pediatría, año 8, t.12, n.2, p.142-176. 1918.

OLIVA, Silvestre.

La protección de la $1^{\mathrm{a}}$ infancia en Buenos Aires: su organización y resultados. Archivos Latinoamericanos de Pediatría, año 7, n.11, p.699645. 1917.

OTERO, Hernán.

El concepto de población en el sistema estadístico nacional. In: Torrado, Susana (Comp.). Población y bienestar en la Argentina del primero al segundo centenario. t.1. Buenos Aires: Edhasa. 2007.

PEREIRA, Júnia Sales.

História de pediatria no Brasil: de final do século XIX a meados do século XX. Tese (Doutorado em História) - Universidade Federal de Minas Gerais, Belo Horizonte. 2006.

PERROT, Michelle.

Figuras y funciones. In: Ariès, Philippe; Duby, Georges. Historia de la vida privada. t.4. Madrid: Taurus. p.127-192. 1989.

PITA, Valeria Silvina.

Administradoras, funcionarios y profesionales en el Hospital de Mujeres Dementes: Buenos Aires, 1880-1990. In: Plotkin, Mariano B.; Zimmermann, Eduardo. Los saberes del Estado. Buenos Aires: Edhasa. p.103-124. 2012.

PLOTKIN, Mariano B.; ZIMMERMANN, Eduardo.

Los saberes del Estado. Buenos Aires: Edhasa. 2012.

PUGA, Teodoro F.

Reseña histórica de la pediatría latinoamericana. Revista de la Sociedad Boliviana de Pediatría, v.46, n.3, p.179-198. 2007.

RÍOS, Julio César; TALAK, A.

La niñez en los espacios urbanos. In: Devoto, Fernando; Madero, Marta (Dir.). Historia de la vida privada en Argentina. t.2. Buenos Aires: Taurus. p.139-161. 1999.

RIVORÊDO, Carlos R.

Pediatria; medicina para crianças? Saúde e Sociedade, n.2. p.33-46. 1998.

RODRÍGUEZ JIMÉNEZ, Pablo. La pediatría en Colombia, 1880-1960: crónica de una alegría. In: Rodríguez Jiménez, Pablo; Manarelli, Emma (Coord.). Historia de la 
infancia en América Latina. Bogotá: Universidad Externado de Colombia. p.359-388. 2007.

RUSTOYBURU, Cecilia.

Pediatría psicosomática y medicalización de la infancia en Buenos Aires, 1940-1970. História, Ciências, Saúde - Manguinhos, v.22, n.4, p.12491265. 2015.

RUSTOYBURU, Cecilia.

Niñez y sexualidad: un análisis histórico de los discursos sobre hormonas en Argentina en los años 1930. In: Cosse, Isabella et al. Infancias, políticas y saberes en Argentina y Brasil, siglos XIX y XX. Buenos Aires: Teseo. p.149-174. 2011.

SOCIÉTÉS...

Sociétés internationales de pédiatrie. Archivos Latinoamericanos de Pediatría, año 3, n.4, p.138143. 1907.

SOPRANO, Germán.

Agencias estatales y procesos de configuración profesional. In: Biernat, Carolina; Ramacciotti,
Karina (Ed.). Historia de la salud y de la enfermedad bajo la lupa de las ciencias sociales. Buenos Aires: Biblos. p.131-146. 2014.

TENTI FANFANI, Emilio.

Estado y pobreza: estrategias típicas de intervención. Buenos Aires: Centro Editor de América Latina. 1990.

TERÁN, Oscar.

Vida intelectual en el Buenos Aires fin-de-siglo, 1880-1910: derivas de la "cultura científica". Buenos Aires: Fondo de Cultura Económica. 2000.

ZAPIOLA, María Carolina.

Los niños entre la escuela, el taller y la calle: Buenos Aires, 1884-1915. Cadernos de Pesquisa, v.39, n.136, p.69-81. 2009.

ZIMMERMAN, Eduardo.

Los liberales reformistas: la cuestión social en Argentina, 1880-1916. Buenos Aires: Sudamericana. 1994. 